\title{
Effect of electronic environment on neutrino-nucleus reactions at r-process sites
}

\author{
F. Minato, ${ }^{1}$ K. Hagino, ${ }^{1}$ N. Takigawa, ${ }^{1}$ A. B. Balantekin,${ }^{2}$ and Ph. Chomaz ${ }^{3}$ \\ 1 Department of Physics, Tohoku University, Sendai 980-8578, Japan \\ ${ }^{2}$ Department of Physics, University of Wisconsin, Madison, Wisconsin 53706 \\ ${ }^{3}$ GANIL-CEA/DSM-CNRS/IN2P3, B.P. 55027, F-14076 Caen Cédex 5, France
}

(Dated: October 16, 2018)

\begin{abstract}
We discuss effects of the electron plasma on charged-current neutrino-nucleus reaction, $\left(\nu_{e}, e^{-}\right)$ in a core-collapse supernova environment. We first discuss the electron screening effect on the final state interaction between the outgoing electron and the daughter nucleus. To this end, we solve the Dirac equation for the outgoing electron with the screened Coulomb potential obtained with the Thomas-Fermi approximation. In addition to the screening effect, we also discuss the Pauli blocking effect due to the environmental electrons on the spectrum of the outgoing electron. We find that both effects hinder the cross section of the charged-current reaction, especially at low incident energies.
\end{abstract}

PACS numbers: $23.40 . \mathrm{Bw}, 26.30 .+\mathrm{k}, 26.50 .+\mathrm{x}, 98.80 . \mathrm{Ft}$

\section{INTRODUCTION}

A large number of neutrinos are emitted from a corecollapse supernova. These neutrinos interact with nuclei through the weak interaction. Although their cross sections are small, it is agreed that their contribution to nucleosynthesis (that is, r-, $\nu$ - and p-processes) is not negligible due to the large neutrino luminosity [1]. Neutrinos may even play a leading role in some cases. For instance, Yoshida et al. recently argued that the abundance ratio between ${ }^{7} \mathrm{Li}$ and ${ }^{11} \mathrm{~B}$ is sensitive to the $\nu$ process and thus can be used to extract information on the neutrino mass hierarchy [2]. Also, the abundance ratio between $\mathrm{U}$ and Th elements, which has been used as a cosmochronometer, may be affected by the $\nu$-process. It is thus important to calculate with high accuracy the cross section of the neutrino-nucleus reactions in a dense star.

In the supernova nucleosynthesis, only the charged current reactions of the electron neutrinos, $\nu_{e}$, and the electron anti-neutrinos, $\bar{\nu}_{e}$, are relevant, since those of $\nu_{\mu}$ and $\nu_{\tau}$ (and their antineutrinos) are suppressed due to the threshold effects. These processes are represented by

$$
\begin{aligned}
\nu_{e}+(Z, A) & \rightarrow e^{-}+(Z+1, A), \\
\bar{\nu}_{e}+(Z, A) & \rightarrow e^{+}+(Z-1, A) .
\end{aligned}
$$

The average energies of $\nu_{e}$ and $\bar{\nu}_{e}$ neutrinos in a core collapse supernova are typically $11 \mathrm{MeV}$ and $16 \mathrm{MeV}$, respectively [3]. It must be emphasized, however, that the exact hierarchy of neutrino energies depend on the details of the underlying microphysics [4]. The outgoing electron and positron feel the Coulomb interaction from the daughter nucleus as they leave. This final state interaction affects the neutrino-nucleus reaction rate $[\underline{5}, 6]$.

In the supernova environment, the motion of the outgoing electron is further perturbed by other charged particles in the environment besides the daughter nucleus, such as protons, electrons, $\alpha$ particles and other heavier nuclei. Such effects have been considered in Ref. [7] for electron capture rates in a dense star. Furthermore, in a high electron density, the reaction (11) is suppressed because low energy electron states are Pauli blocked. It is crucial to take into account those two effects in order to accurately estimate the neutrino-nucleus reaction rate for nucleosynthesis.

In this paper, we perform such calculations, taking into consideration both the electron screening and Pauli blocking effects. For this purpose, we use the ThomasFermi theory for electron screening, which has often been used in many-body physics. We then calculate the cross section of a neutrino-nucleus reaction using the distorted wave Born approximation (DWBA) method, including the screening and the Pauli blocking effects simultaneously.

The paper is organized as follows. In Sec. II, we detail our model for the charged current neutrinonucleus reaction in a supernova environment, that uses the Thomas-Fermi theory for electron screening, and the DWBA method with the Pauli blocking effect. In Sec. III, we present the results of our calculation for the ${ }^{56} \mathrm{Fe}\left(\nu_{e}, e^{-}\right){ }^{56} \mathrm{Co}$ and ${ }^{208} \mathrm{~Pb}\left(\nu_{e}, e^{-}\right){ }^{208} \mathrm{Bi}$ reactions, and discuss the effects of the electronic environment. We summarize our paper in Sec. IV.

\section{NEUTRINO-NUCLEUS REACTIONS AT FINITE ELECTRON DENSITY}

\section{A. Electron screening effect}

We first discuss the electron screening effect on the final state interaction between the outgoing electron and the daughter nucleus. Despite of the charge neutral condition of the environment, the screening effect can still be significant because the daughter nucleus attracts the environmental electrons and polarizes the background charge distribution. The positive charge particles are relatively heavy, and thus we neglect its polarization.

We assume that the electron charge distribution is ho- 
mogeneous with density $\rho_{e}^{0}$ in the absence of the daughter nucleus. This charge distribution of the environmental electrons is modified to $\rho_{e}(\boldsymbol{r})$ due to the presence of the daughter nucleus, whose charge distribution is represented by $e \rho_{\mathrm{N}}(\boldsymbol{r})$. Neglecting the the interaction among the environmental electrons, which is valid at high temperatures, the Coulomb field $\phi(\boldsymbol{r})$ at $\boldsymbol{r}$ from the daughter nucleus reads

$$
\phi(\boldsymbol{r})=\int d \boldsymbol{r}^{\prime} \frac{e \rho_{\mathrm{N}}\left(\boldsymbol{r}^{\prime}\right)-e \delta \rho_{e}\left(\boldsymbol{r}^{\prime}\right)}{\left|\boldsymbol{r}-\boldsymbol{r}^{\prime}\right|},
$$

where $\delta \rho_{e}(\boldsymbol{r}) \equiv \rho_{e}(\boldsymbol{r})-\rho_{e}^{0}$ is the polarization charge. In order to evaluate this function, we assume a sharp-cut charge distribution for $\rho_{\mathrm{N}}$, that is, $\rho_{\mathrm{N}}(\boldsymbol{r})=\left[3 Z /\left(4 \pi R^{3}\right)\right]$. $\theta(R-r)$ with a nuclear radius of $R$. Here, $Z$ is the atomic number of the daughter nucleus.

For the electron density $\rho_{e}$, we use the Thomas-Fermi theory. The polarization charge then reads

$$
\delta \rho_{e}(\boldsymbol{r})=\frac{1}{3 \pi^{2} \hbar^{3}}\left(2 m \epsilon_{F}(\boldsymbol{r})\right)^{3 / 2}-\rho_{e}^{0} .
$$

Here, $m$ is the electron mass, and the local Fermi energy $\epsilon_{F}(\boldsymbol{r})$ is given by

$$
\epsilon_{F}(\boldsymbol{r})=\epsilon_{F}^{0}+e \phi(\boldsymbol{r}),
$$

with $\epsilon_{F}^{0}=\left(3 \pi^{2} \hbar^{3} \rho_{e}^{0}\right)^{2 / 3} / 2 m$. We have assumed that atoms are completely ionized, without any bound electrons.

Since the electron density $\rho_{e}$ depends explicitly on the Coulomb field $\phi$, we evaluate Eq. (3) self-consistently, assuming the spherical symmetry. The boundary condition is imposed so that the Coulomb potential vanishes at the radius where the net negative charge inside is equal to the charge number of the daughter nucleus.

Once the Coulomb field $\phi$ is obtained, we solve the Dirac equation for the outgoing election with the potential $V_{C}(r)=-e \phi(r)$. Writing the wave function of the outgoing electron as,

$$
\psi_{j l m}^{E}(\boldsymbol{r})=\frac{1}{r}\left(\begin{array}{c}
i P_{j l}^{E}(r) \mathcal{Y}_{j l m}(\hat{\boldsymbol{r}}) \\
Q_{j l}^{E}(r) \mathcal{Y}_{j, 2 j-l, m}(\hat{\boldsymbol{r}})
\end{array}\right),
$$

with

$$
\mathcal{Y}_{j l m}(\hat{\boldsymbol{r}})=\sum_{m_{l}, m_{s}}\left\langle l m_{l} 1 / 2 m_{s} \mid j m\right\rangle Y_{l m_{l}}(\hat{\boldsymbol{r}}) \chi_{m_{s}},
$$

where $\chi_{m_{s}}$ is the two component spin wave function, the Dirac equation reads

$$
\begin{aligned}
& \frac{d P_{j l}^{E}}{d r}=-\frac{\kappa}{r} P_{j l}^{E}(r)-\left[E+m-V_{c}(r)\right] Q_{j l}^{E}(r), \\
& \frac{d Q_{j l}^{E}}{d r}=\frac{\kappa}{r} Q_{j l}^{E}(r)+\left[E+m-V_{c}(r)\right] P_{j l}^{E}(r),
\end{aligned}
$$

where $\kappa=(l-j)(2 j+1)$. We use the computer code RADIAL [8] to solve the Dirac equation. We use these wave functions in Sec. II C in order to compute the cross sections of charged current neutrino-nucleus reactions.

\section{B. Pauli blocking effect}

In the high density limit, the environmental electrons are almost degenerate and the occupation probability of the lowest energy states is close to unity. In this situation, the production of a low energy electron is suppressed due to the Pauli blocking effect of the environmental electrons. For a given electron density $\rho_{e}^{0}$, the Fermi energy $\mu_{e}$ can be estimated using the relativistic Fermi gas model,

$$
\rho_{e}^{0}=\frac{m^{3} c^{6}}{\pi^{2} \hbar^{3}} \int_{0}^{\infty} \frac{\sinh ^{2} x \cosh x}{\exp \left(\beta\left(m c^{2} \cosh x-\mu_{e}\right)\right)+1} d x,
$$

where $\cosh x=p / m c^{2}, p$ being the momentum of the electron. In order to take into account the Pauli blocking effect, we multiply a factor $\left(1-f_{e}\left(E_{e}, T_{e}, \mu_{e}\right)\right)$ to the cross section, where $E_{e}$ is the energy of the outgoing electron and $f_{e}$ is the distribution function of the environmental electrons given by

$$
f_{e}\left(E, T_{e}, \mu_{e}\right)=\frac{1}{1+\exp \left[\left(E-\mu_{e}\right) / T_{e}\right]} .
$$

\section{Cross sections}

We estimate the cross sections of the neutrino-nucleus reactions using the DWBA method, which is the most natural way to include the screening correction. This method in fact improves methods typically used to calculate final state interactions, such as so-called Fermi function and effective momentum approximations [9]. The cross sections for the Fermi type transition from the ground state of a parent nucleus with $J_{i}^{\pi}=0^{+}$to the 
$J^{\pi}$ state in the daughter nucleus is given by [9],

$$
\begin{gathered}
\frac{d \sigma}{d E_{e}}=G^{2} \frac{E_{e}+k_{e}}{2 k_{e}}\left(1-f_{e}\left(E_{e}, T_{e}, \mu_{e}\right)\right) \\
\times \sum_{j, L}\left(\begin{array}{c}
2 j+1)(2 L+1) \\
\times\left(\begin{array}{l}
\left\langle j-\frac{1}{2} 0, L 0 \mid J 0\right\rangle^{2}\left(F_{L J, j}^{-2}+G_{L J, j}^{+2}\right) \\
+\left\langle j+\frac{1}{2} 0, L 0 \mid J 0\right\rangle^{2}\left(F_{L J, j}^{+2}+G_{L J, j}^{-2}\right)
\end{array}\right) \\
-2\left\langle j+\frac{1}{2} 0, L 0 \mid J 0\right\rangle \\
\left(\begin{array}{l}
\sqrt{\left[J^{2}-\left(L+\frac{1}{2}-j\right)^{2}\right]} \\
\times \sqrt{\left[(J+1)^{2}-\left(L+\frac{1}{2}-j\right)^{2}\right]} \\
\times\left\langle j-\frac{1}{2} 0, L+10 \mid J 0\right\rangle \\
\times\left(F_{L+1 J, j}^{-} G_{L J, j}^{-}-F_{L J, j}^{+} G_{L+1 J, j}^{+}\right) \\
+\sqrt{\left[J^{2}-\left(L+\frac{1}{2}+j\right)^{2}\right]} \\
\times \sqrt{\left[(J+1)^{2}-\left(L+\frac{1}{2}+j\right)^{2}\right]} \\
\times\left\langle j-\frac{1}{2} 0, L-10 \mid J 0\right\rangle \\
\times\left(F_{L-1 J, j}^{-} G_{L J, j}^{-}-F_{L J, j}^{+} G_{L-1 J, j}^{+}\right)
\end{array}\right) \\
\times
\end{array}\right),
\end{gathered}
$$

where $G$ is the Fermi constant. $E_{e}$ and $k_{e}$ are the energy and momentum of the outgoing electron, respectively, and $k_{\nu}$ is the incident neutrino momentum. The relation between the neutrino energy $E_{\nu}$ and the electron energy $E_{e}$ is $E_{\nu}=\hbar k_{\nu} c=E_{\mathrm{x}}+E_{e}-Q$, where $E_{\mathrm{x}}$ is the excitation energy for the nuclear transition with respect to the ground state of the parent nucleus, and $Q=m_{n} c^{2}-m_{p} c^{2}$ is the mass difference between neutron and proton. $F_{L J, j}^{ \pm}$and $G_{L J, j}^{ \pm}$are defined as [9],

$$
\begin{aligned}
F_{L J, j}^{ \pm} & =\int d \boldsymbol{r} \frac{P_{j, j \pm 1 / 2}^{E_{e}}(r)}{r} j_{L}\left(k_{\nu} r\right) Y_{J M}(\hat{\boldsymbol{r}}) \rho_{f i}(\boldsymbol{r})(13) \\
G_{L J, j}^{ \pm} & =\int d \boldsymbol{r} \frac{Q_{j, j \pm 1 / 2}^{E_{e}}(r)}{r} j_{L}\left(k_{\nu} r\right) Y_{J M}(\hat{\boldsymbol{r}}) \rho_{f i}(\boldsymbol{r})(14)
\end{aligned}
$$

respectively, $\rho_{f i}(\boldsymbol{r})$ being the transition density for the nuclear Fermi transition.

\section{RESULTS}

We now evaluate numerically the electron screening and the Pauli blocking effects on the charged current ${ }^{56} \mathrm{Fe}\left(\nu_{e}, e^{-}\right)^{56} \mathrm{Co}$ and ${ }^{208} \mathrm{~Pb}\left(\nu_{e}, e^{-}\right)^{208} \mathrm{Bi}$ reactions. We set the electron temperature to be $T_{e}=0.5 \mathrm{MeV}$. We consider the Fermi type transition to the $J^{\pi}=0^{+}$state at $E_{\mathrm{x}}=3.5 \mathrm{MeV}$ in ${ }^{56} \mathrm{Co}$ 12 and $E_{\mathrm{x}}=15.0 \mathrm{MeV}$

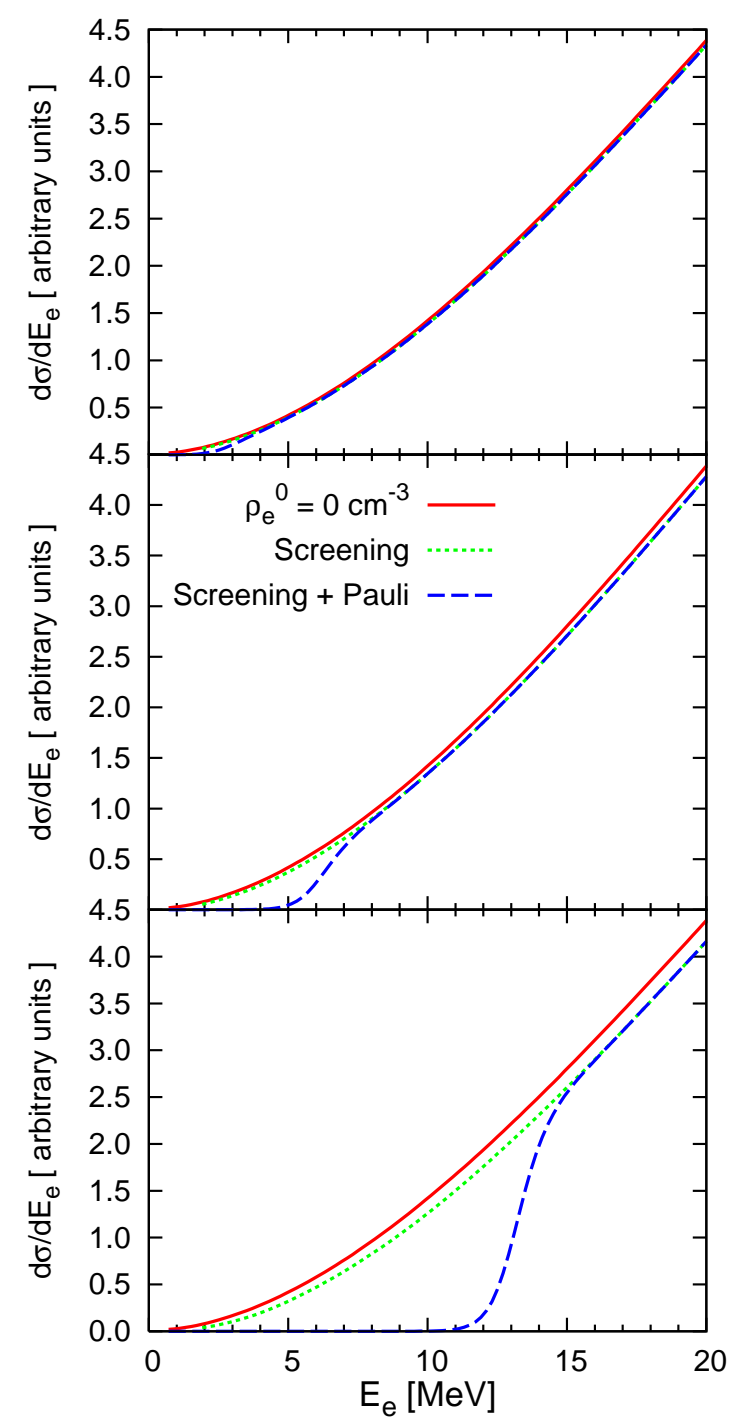

FIG. 1: (Color online) The cross sections for the charged current $\nu_{e}+{ }^{56} \mathrm{Fe} \rightarrow e^{-}+{ }^{56} \mathrm{Co}$ reaction as a function of the energy of the outgoing electron for three densities of the environmental electrons, $\rho_{e}^{0}$. The Fermi transition to the $J^{\pi}=0^{+}$state at $E_{x}=3.5 \mathrm{MeV}$ in ${ }^{56} \mathrm{Co}$ is considered. The top, middle, and bottom panels are for $\rho_{e}^{0}=10^{32}, 10^{33}$, and $10^{34} \mathrm{~cm}^{-3}$, respectively. The solid line shows the results in the absence of the environmental electrons, while the dotted line includes the electron screening effects. The dashed line takes into account both the screening and the Pauli blocking effects.

in ${ }^{208} \mathrm{Bi}$ [1]. For simplicity, we follow Ref. [9] and assume the transition density which is proportional to $\rho_{f i} \propto \delta(r-R) Y_{J M}(\theta, \phi)$.

The differential cross sections $d \sigma / d E_{e}$ for the ${ }^{56} \mathrm{Fe}\left(\nu_{e}, e^{-}\right){ }^{56} \mathrm{Co}$ and the ${ }^{208} \mathrm{~Pb}\left(\nu_{e}, e^{-}\right){ }^{208} \mathrm{Bi}$ reactions are shown in Figs. 1 and 2 , respectively. The solid line shows the results in the absence of the environmental electrons. The top, middle and bottom panels are for 


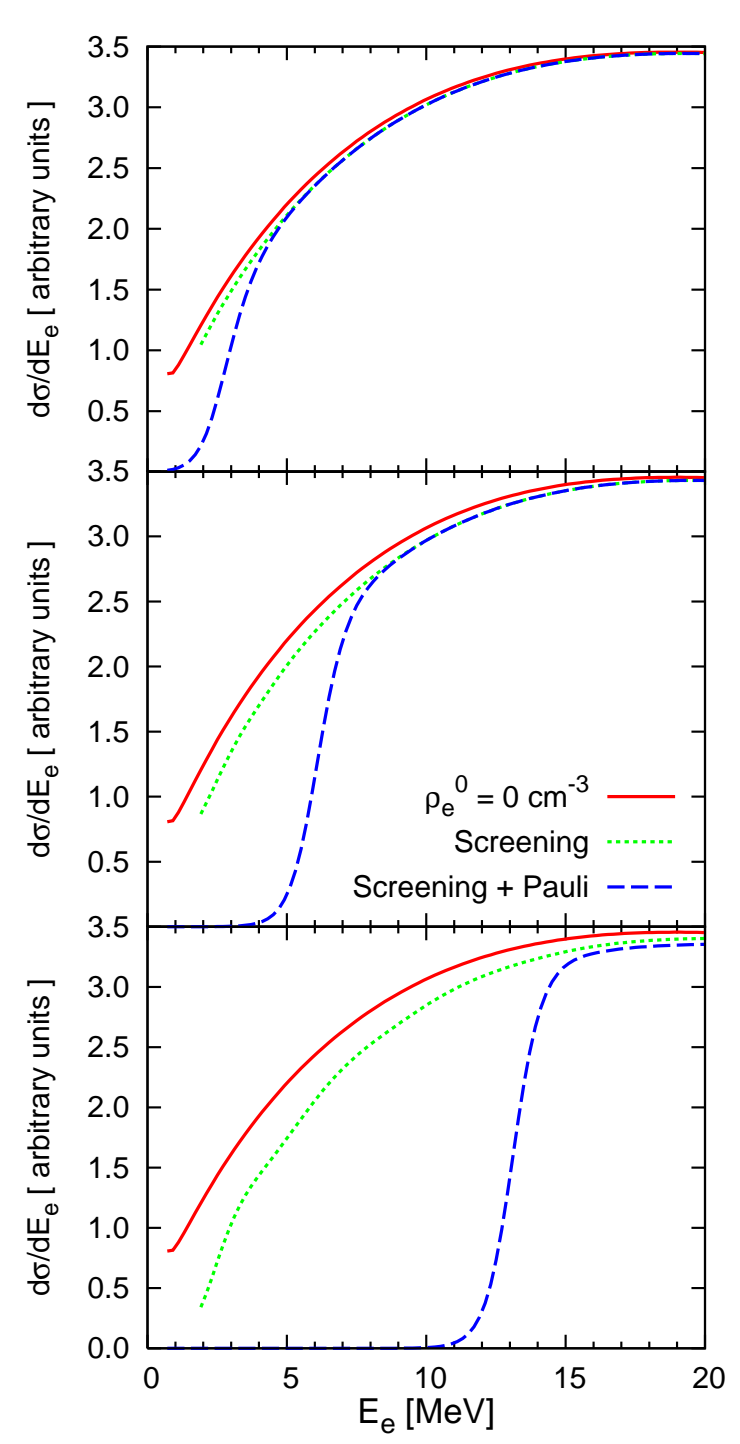

FIG. 2: (Color online) The same as Fig. 1, but for the $\nu_{e}+{ }^{208} \mathrm{~Pb} \rightarrow e^{-}+{ }^{208} \mathrm{Bi}$ reaction. The Fermi transition to the $J^{\pi}=0^{+}$state at $E_{x}=15.0 \mathrm{MeV}$ in ${ }^{208} \mathrm{Bi}$ is considered.

the electron density of $\rho_{e}^{0}=10^{32}, 10^{33}$, and $10^{34} \mathrm{~cm}^{-3}$, respectively. The dotted line denotes the results with the electron screening effects, while the dashed line takes into account both the screening and the Pauli blocking effects. For the electron density smaller than $10^{31} \mathrm{~cm}^{-3}$, we find that both the effects are marginal. The screening effect is larger in the ${ }^{208} \mathrm{~Pb}\left(\nu_{e}, e^{-}\right)^{208} \mathrm{Bi}$ than in the ${ }^{56} \mathrm{Fe}\left(\nu_{e}, e^{-}\right)^{56} \mathrm{Co}$ reaction, as is expected. The electron Fermi energy obtained with Eq. (10), for $\rho_{e}^{0}=10^{32}, 10^{33}$, and $10^{34} \mathrm{~cm}^{-3}$ is $2.59,5.99$, and $13.1 \mathrm{MeV}$, respectively. We see from the figures that the Pauli blocking effects are important below these energies. We have confirmed that the screening and the blocking effects disappear in the high $E_{e}$ limit.

For the anti-neutrino induced reactions, (2), the screening effect increases the cross section in contrast to the neutrino induced reactions shown in Figs. 1 and 2, while the Pauli blocking effect is irrelevant in the absence of a positron background. The net effect of the environmental electrons thus increases the cross sections for these reactions.

We next discuss total cross sections. In order to compute the total cross sections, we integrate the differential cross sections, Eq. (12), with a weight factor given by the energy distribution for the incident neutrino, $n_{\nu}\left(E_{\nu}\right)$. That is,

$$
\sigma=\int_{0}^{\infty} \frac{d \sigma}{d E_{\nu}} n_{\nu}\left(E_{\nu}\right) d E_{\nu}
$$

Following Ref. [10], we assume that the distribution function $n_{\nu}$ is given by,

$$
n_{\nu}\left(E_{\nu}\right)=\frac{1}{F T_{\nu}^{3}} \frac{E_{\nu}^{2}}{e^{E_{\nu} / T_{\nu}}+1}
$$

with

$$
F=\int_{0}^{\infty} d E \frac{E^{2}}{e^{E}+1}
$$

at distances outside a neutrino sphere, where r-process is considered to take place. Here $T_{\nu}$ is the neutrino temperature at the neutrino sphere.

Figures 3 and 4 show the total cross sections for the ${ }^{56} \mathrm{Fe}\left(\nu_{e}, e^{-}\right)^{56} \mathrm{Co}$ and the ${ }^{208} \mathrm{~Pb}\left(\nu_{e}, e^{-}\right){ }^{208} \mathrm{Bi}$ reactions as a function of the density of the environmental electrons, respectively. These are plotted as the ratio to the total cross sections in the absence of the environmental electrons, $\sigma_{0}$. The neutrino temperature $T_{\nu}$ in Eq. (16) is set to be $4 \mathrm{MeV}$. The dotted line takes into account only the screening effects, while the dashed line includes both the screening and the Pauli blocking effects. We see that the Pauli blocking effect influences the ${ }^{208} \mathrm{~Pb}\left(\nu_{e}, e^{-}\right)^{208} \mathrm{Bi}$ reaction much more significantly than the ${ }^{56} \mathrm{Fe}\left(\nu_{e}, e^{-}\right){ }^{56} \mathrm{Co}$ reaction. This is due to the fact that the neutrino energy distribution $n_{\nu}$ has a peak around $E_{\nu}=8.87 \mathrm{MeV}$ at the neutrino temperature considered here. For the former reaction, the excitation energy $E_{x}$ is $15 \mathrm{MeV}$ and the energy of the produced electron is always small, for which the blocking effect is important. For the latter reaction, on the other hand, the excitation energy is relatively small $\left(E_{x}=3.5 \mathrm{MeV}\right)$, and the average energy of the outgoing electron is above the electron Fermi energy unless the electron density $\rho_{e}^{0}$ is large.

We find that the screening effect on the total cross sections is sensitive to the variation of the Coulomb potential due to the environmental electrons. For instance, the depth of the potential between the outgoing electron and ${ }^{208} \mathrm{Bi}$ is $25.2 \mathrm{MeV}$ in the absence of the environmental electrons. At $\rho_{e}^{0}=10^{31}, 10^{32}$ and $10^{31} \mathrm{~cm}^{-3}$, it is 25.08 , 24.97, and $24.73 \mathrm{MeV}$, respectively. The corresponding change of the total cross section is $2.25,4.48$, and $10.1 \%$, respectively. Clearly, it is important to estimate carefully the Coulomb potential in the high electron density region. 


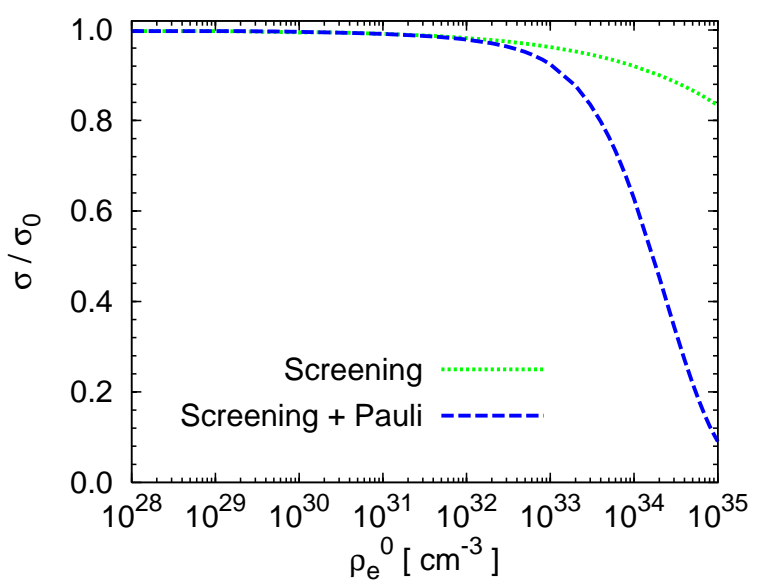

FIG. 3: (Color online) The total cross sections for the $\nu_{e}+{ }^{56} \mathrm{Fe} \rightarrow e^{-}+{ }^{56} \mathrm{Co}$ reaction as a function of the density of the environmental electrons. These are given as the ratio to the cross sections in the absence of the environmental electrons, $\sigma_{0}$. The meaning of each line is the same as in Fig. 1 .

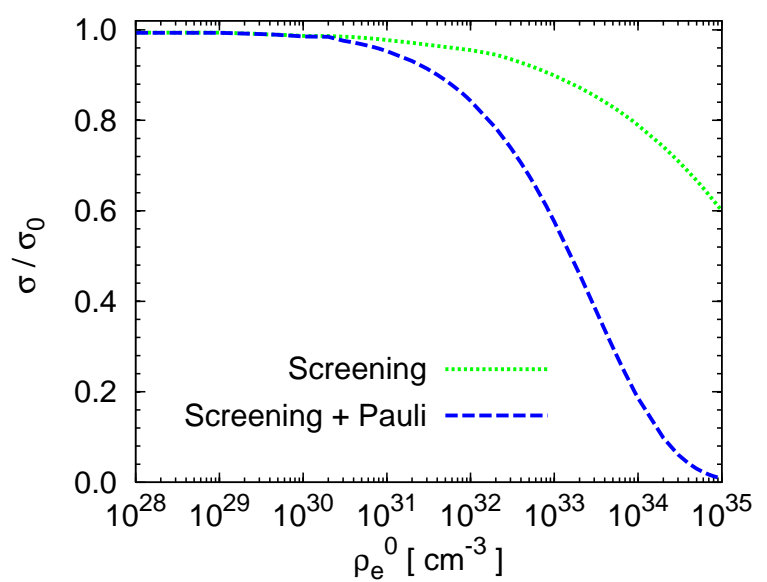

FIG. 4: (Color online) The same as Fig. 3, but for the $\nu_{e}+{ }^{208} \mathrm{~Pb} \rightarrow e^{-}+{ }^{208} \mathrm{Bi}$ reactions.

\section{SUMMARY}

The motion of the outgoing electron in the final state of a charged current neutrino-nucleus reaction is affected by surrounding environmental electrons in a supernova. We have discussed the electron screening as well as the Pauli blocking effects due to the environmental electrons on cross sections of the neutrino-nucleus reaction. For this purpose, we used the Thomas-Fermi theory for the screening potential, and the DWBA with the Pauli blocking factor for the cross sections. Our results for the ${ }^{208} \mathrm{~Pb}\left(\nu_{e}, e^{-}\right){ }^{208} \mathrm{Bi}$ and ${ }^{56} \mathrm{Fe}\left(\nu_{e}, e^{-}\right){ }^{56} \mathrm{Co}$ reactions show that both the effects hinder the cross sections, especially at high electron densities. We have also shown that the Pauli blocking effect is more significant in the former reaction than in the latter reaction due to the larger excitation energy for the Fermi transition. The screening effect is also larger in the former reaction because of the larger atomic number.

As far as we know, the screening and the Pauli blocking effects discussed in this paper have not been taken into account in the calculations for r-process nucleosynthesis. It would be of intriguing to see how these effects influence the r-process nucleosynthesis.

\section{Acknowledgment}

We thank Y. Kato for useful discussions on electron screening. We also acknowledge the 21st Century for Center of Excellence (COE) Program "Exploring New Science by Bridging Particle-Matter Hierarchy" at Tohoku University. Discussions during the 4th COE symposium were useful to complete this work. This work was supported in part by the Grant-in-Aid for Scientific Research, Contract No. 16740139 from the Japanese Ministry of Education, Culture, Sports, Science, and Technology, in part by the U.S. National Science Foundation Grant No. PHY-0555231, and in part by the University of Wisconsin Research Committee with funds granted by the Wisconsin Alumni Research Foundation.
[1] A. B. Balantekin and G. M. Fuller, J. Phys. G 29, 2513 (2003).

[2] T. Yoshida, T. Kajino, H. Yokomakura, K. Kimura, A. Takamura and Dieter H. Hartmann, Phys. Rev. Lett. 96, 091101 (2006).

[3] Y.-Z. Qian, G.M. Fuller, G.J. Mathews, R.W. Mayle, J.R. Wilson, S.E. Woosley, Phys. Rev. Lett. 71, 1965 (1993).

[4] S. Hannestad and G. Raffelt, Astrophys. J. 507, 339 (1998); R. Buras, H.T. Janka, M.T. Keil, G.G. Raffelt, and M. Rampp, Astrophys. J. 587, 320 (2003); A. B. Balantekin and H. Yuksel, New J. Phys. 7, 51 (2005).
[5] J. D. Walecka, Theoretical Nuclear and Subnuclear Physics (Oxford University Press, New York, 1995), Sec. 45.

[6] M. Morita, Beta decay and muon capture (W.A. Benjamin Inc., Reading, 1973).

[7] N. Itoh, N. Tomizawa, M. Tamamura and S. Wanajo, Astrophys. J. 579, 380 (2002).

[8] F. Salvat, J.M. Fernández-Varea, and W. Williamson Jr., Comp. Phys. Comm. 90, 151 (1995).

[9] J. Engel, Phys. Rev. C 57, 2004 (1998).

[10] Y.-Z. Qian and G. M. Fuller, Phys. Rev. D 51, 1479 (1995). 
[11] A. Krasznahorkay, H. Akimune, M. Fujiwara et. al., A403, 317 (1983).

[12] T. Murakami, S. Nishihara and T. Nakagawa, Nucl. Phys. 